\title{
Tight RMR Lower Bounds for Mutual Exclusion and Other Problems.
}

\author{
(Extended Abstract)
}

\author{
Hagit Attiya \\ Department of Comp. Sci. \\ Technion \\ hagit@cs.technion.ac.il
}

\author{
Danny Hendler \\ Department of Comp. Sci. \\ Ben-Gurion University \\ hendlerd@cs.bgu.ac.il
}

\author{
Philipp Woelfel \\ Department of Comp. Sci. \\ University of Calgary \\ woelfel@cpsc.ucalgary.ca
}

\begin{abstract}
We investigate the remote memory references (RMRs) complexity of deterministic processes that communicate by reading and writing shared memory in asynchronous cache-coherent and distributed shared-memory multiprocessors.

We define a class of algorithms that we call order encoding. By applying information-theoretic arguments, we prove that every order encoding algorithm, shared by $n$ processes, has an execution that incurs $\Omega(n \log n)$ RMRs. From this we derive the same lower bound for the mutual exclusion, bounded counter and store/collect synchronization problems. The bounds we obtain for these problems are tight. It follows from the results of [10] that our lower bounds hold also for algorithms that can use comparison primitives and loadlinked/store-conditional in addition to reads and writes. Our mutual exclusion lower bound proves a longstanding conjecture of Anderson and Kim.
\end{abstract}

\section{Categories and Subject Descriptors}

D.1.3 [Software]: Programming Techniques-Concurrent programming; F.1.2 [Theory of Computation]: Computation by Abstract Devices-Modes of Computation [Parallelism and concurrency]; F.2.2 [Theory of Computation]: Analysis of Algorithms and Problem Complexity_Nonnumerical Algorithms and Problems

\section{General Terms}

Algorithms, Theory

\section{Keywords}

information theory, shared-memory, lower bound techniques, mutual exclusion, bounded counter, store/collect object

\footnotetext{
*The first author is supported in part by the Israel Science Foundation (grant number 953/06).
}

\footnotetext{
Permission to make digital or hard copies of all or part of this work for personal or classroom use is granted without fee provided that copies are not made or distributed for profit or commercial advantage and that copies bear this notice and the full citation on the first page. To copy otherwise, to republish, to post on servers or to redistribute to lists, requires prior specific permission and/or a fee.

STOC'08, May 17-20, 2008, Victoria, British Columbia, Canada.

Copyright 2008 ACM 978-1-60558-047-0/08/05 ...\$5.00.
}

\section{INTRODUCTION}

The memory hierarchy in shared-memory multi-processor systems places some of the memory locally, while the rest of the memory is remote, e.g., in other processing units or in dedicated storage. For example, in cache-coherent (CC) systems, each processor maintains local copies of shared variables in its cache; the consistency of copies in different caches is ensured by a coherence protocol. At any given time, a variable is local to a process if the coherence protocol guarantees that the corresponding cache contains an up-to-date copy of the variable, and is remote otherwise. In distributed sharedmemory (DSM) systems, on the other hand, each shared variable is permanently locally accessible to a single processor and remote to all other processors.

References to remote memory are orders of magnitude slower than accesses to the local memory module; they generate traffic on the processor-to-memory interconnect, which can be a bottleneck and cause further slow-down. For these reasons, the performance of many algorithms for shared memory multiprocessor systems depends critically on the number of references to remote memory they generate $[7,14]$.

The remote memory references complexity measure, abbreviated $R M R$ cost, charges an algorithm with one unit for each access of remote memory, namely, reading from or writing to memory locations that do not reside locally at the process; writing, reading or even spinning on a memory location that is locally available is considered free. The RMR cost is considered to accurately predict the actual performance of a concurrent algorithms deployed in a multiprocessing system.

The familiar way to measure time complexity, by counting the total number of shared memory accesses, regardless of whether they are local or remote, is inadequate for blocking problems such as mutual exclusion: For such problems, a process may perform an unbounded number of memory accesses while performing a local spin loop, waiting for another process [1]. Instead, recent mutual exclusion work mostly uses the RMR cost to evaluate algorithms (see, e.g., $[2,5,6,13])$.

We prove tight lower bounds on the RMR cost of key synchronization problems-mutual exclusion, bounded counters, and store/collect.

Our Technique and Contributions. We define order encoding algorithms. Roughly speaking, an algorithm is order encoding if, for each of its executions $E$, the order in which the operations that were performed in $E$ "took effect" can be determined based on the state of the shared memory after $E$ terminates.

Our key technical result (Theorems 1 and 9) establishes that every $n$-process order encoding algorithm has an execution that incurs 
$\Omega(n \log n)$ RMRs. To prove this, we construct a set $\mathcal{E}$ of $n$ ! stylized executions of the order encoding algorithm. The set $\mathcal{E}$ has the properties that (a) different executions of $\mathcal{E}$ result in different sharedmemory states, and (b) each execution $E \in \mathcal{E}$ can be represented by a bit-string whose length is bounded from above (up to a constant factor) by the total number of RMRs performed in $E$. Since $|\mathcal{E}|=n$ !, a simple information theoretic argument shows that the RMR cost of at least one of the executions of $\mathcal{E}$ is $\Omega(n \log n)$.

The reader may wonder whether an algorithm implementing one of the problems we consider, e.g. a mutual exclusion algorithm, must be order encoding: an execution of a mutual exclusion algorithm does not necessarily record the order of its operations in shared memory! Indeed, we do not argue directly about the algorithms for the problems we consider. Rather, we present "wrappers" to obtain corresponding order encoding algorithms.

The mutual exclusion problem is at the core of distributed computing theory [16]. Using the technique described above, we prove a lower bound of $\Omega(n \log n)$ RMRs on any implementation of mutual exclusion from reads and writes (Theorem 11). This bound, as well as all other bounds we present, holds for both the CC and the DSM models. This result confirms a longstanding conjecture of Anderson and Kim [4]. We then prove a similar result for bounded counters (Theorem 13) and for store/collect objects (Theorem 15).

In [10] it is proven that any CC or DSM algorithm using read and write operations, load-linked/store-conditional (LL/SC), and comparison primitives, can be simulated by an algorithm that uses only read and write operations, with just a constant blowup in the RMR complexity. It follows that the lower bounds hold also if the algorithms can use comparison primitives and LL/SC in addition to reads and writes.

Related Work. Considerable research has focused on proving RMR bounds for the mutual exclusion problem. Anderson and Yang presented an $O(\log n)$ RMRs mutual exclusion algorithm [17]. Our results establish that this is the best possible. Anderson and Kim proved a lower bound of $\Omega(\log n / \log \log n)$ on the RMR complexity of mutual exclusion algorithms that use reads and writes only [2], improving on a previous lower bound of $\Omega(\log \log n / \log \log \log n)$ obtained by Cypher [8].

Fan and Lynch [9] proved an $\Omega(n \log n)$ lower bound on the state change cost of mutual exclusion. This does not imply a corresponding RMR lower bound for either the CC or the DSM models. Nevertheless, their technique introduced several novel ideas, which our technique borrows and extends.

For lack of space, we refer the interested reader to [3] for a comprehensive discussion of RMR upper and lower bounds.

\section{MODEL}

Our model of computation is based on [12]. A concurrent system models an asynchronous shared memory system where $n$ deterministic processes communicate by executing operations on shared variables.

Each process is a sequential execution path that performs a sequence of steps, each of which invokes a single operation on a single variable and receives a corresponding response. The operations allowed in our model are read, write, comparison primitives $[2,10]$ (a.k.a. conditional operations) and load-linked/store-conditional. If step $\sigma$ invokes an operation on variable $v$ we say that $\sigma$ accesses $v$. A variable that supports the read and write operations only is called a register.

In this paper we consider the cache-coherent (CC) and the distributed shared-memory (DSM) computation models. Each processor in a CC machine maintains local copies of shared variables inside its cache, whose consistency is ensured by a coherence protocol. At any given time a variable is remote to a processor if the corresponding cache does not contain an up-to-date copy of the variable.

Our lower bounds apply to both families of CC coherence algorithms: write-through and write-back [15]. Quoting from [10]: "In a write-through protocol, to read a register $R$ a process $p$ must have a (valid) cached copy of $R$. If it does, $p$ reads that copy without causing an RMR; otherwise, $p$ causes an RMR that creates a cached copy of $R$. To write $R, p$ causes an RMR that invalidates (i.e., effectively deletes) all other cached copies of $R$ and writes $R$ to main memory.

In a write-back protocol, each cached copy is held in either "shared" or "exclusive" mode. To read a register $R$, a process $p$ must hold a cached copy of $R$ in either mode. If it does, $p$ reads that copy without causing an RMR. Otherwise, $p$ causes an RMR that: (a) eliminates any copy of $R$ held in exclusive mode, typically by downgrading the status to shared and, if the exclusive copy was modified, writing $R$ back to memory; and (b) creates a cached copy of $R$ held in shared mode. To write $R, p$ must have a cached copy of $R$ held in exclusive mode. If it does, $p$ writes that copy without causing RMRs. Otherwise, $p$ causes an RMR that: (a) invalidates all other cached copies of $R$ and writes any modified copy held in exclusive mode back to memory; and (b) creates a cached copy of $R$ held in exclusive mode."

In the DSM model, each processor owns a segment of shared memory that can be locally accessed without traversing the processor-to-memory interconnect. Thus, every register is (forever) local to a single processor and remote to all others. An access of a remote register is an RMR. For simplicity and without loss of generality, we assume that each of the processes participating in the algorithms we consider runs on a unique processor.

We say that a step $\sigma$ by process $p$ that accesses a variable $v$ is local if $\sigma$ can be applied on a local copy of $v$, without using the processor-to-memory interconnect. Otherwise we say that $\sigma$ is a remote memory reference (RMR).

An execution is a sequence of steps performed by processes as they execute their algorithms. A process $p$ is idle after an execution $E$ if, right after $E, p$ is not in the midst of executing its algorithm; that is, either $p$ did not start performing its algorithm in $E$ or $p$ terminates its algorithm in $E$.

\section{LOWER BOUND IN THE CC MODEL}

The algorithms we consider in the following only use read and write operations. However, by the results of [10], all our lower bounds hold also for algorithms that can use also comparison operations and LL/SC.

\subsection{Order Encoding Algorithms and the Main Theorem}

We consider a set $\mathcal{P}=\left\{p_{1}, \ldots, p_{n}\right\}$ of $n$ processes, where each process $p_{i}$ executes algorithm $A_{i}$. Our proof technique assumes that the algorithms $A_{i}$ may invoke the following two types of special operations, in addition to reads and writes: A record-operation, performed by $p_{i}$, receives an arbitrary value as input and records it by writing it to a register that is only accessible to $p_{i}$. An orderoperation, by process $p_{i}$, is a read from a register $R_{i}^{*}$, that is only accessible by $p_{i}$. We use order-operations to determine the order in which these operations are executed during the execution, in a way that is made precise later. The last operation performed by each algorithm $A_{i}$ is a record-operation. This is the only record-operation performed by $A_{i}$. $A_{i}$ is also required to perform the order-operation exactly once.

We emphasize that the above special operations do not limit the set of algorithms to which our lower bounds apply. They are only 
inserted to wrapper versions of these algorithms and our technique is applied to these wrapper versions.

Let $\mathcal{E}$ be the set of all executions in which each process $p_{i}$ either does not participate or executes its algorithm $A_{i}$ exactly once. For an execution $E \in \mathcal{E}$, we write $p_{i} \prec_{E} p_{j}$ if process $p_{i}$ executes its orderoperation in $E$ before process $p_{j}$ does. If all $n$ processes complete their algorithms in $E$ then $\prec_{E}$ is a total order. The record-vector of an execution $E$ is the vector $\vec{R}[E]=\left(\gamma_{1}, \ldots, \gamma_{n}\right)$, where $\gamma_{i}$ is the value recorded by process $p_{i}$ in its last step in $E ; \gamma_{i}$ is denoted $R_{i}[E]$.

Let $L=\left(q_{1}, \ldots, q_{k}\right)$ be a list of distinct processes in $\mathcal{P}$. The $L$-solo execution, denoted $E_{\text {sol }}(L) \in \mathcal{E}$, is the execution in which the set of participating processes is $\left\{q_{1}, \ldots, q_{k}\right\}$ and, for $1 \leq i<k$, process $q_{i}$ terminates its algorithm before process $q_{i+1}$ executes its first step. If $L$ is a permutation $\pi \in S_{n}$, we simply write $L_{\pi}$.

DEFINITION 1. A sequence of algorithms $\left(A_{1}, \ldots, A_{n}\right)$ is called "order encoding" if for all permutations $\pi \in S_{n}$, for all lists $L=$ $\left(p_{\pi(1)}, \ldots, p_{\pi(k)}\right)$ of length $k, 1 \leq k \leq n$, and for all executions $E$ in which exactly the processes $p_{\pi(1)}, \ldots, p_{\pi(k)}$ participate and terminate, the following two properties hold:

(a) If there are $1 \leq i<j \leq k$, such that $p_{\pi(j)} \prec_{E} p_{\pi(i)}$, then $\vec{R}[E] \neq$ $\vec{R}\left[E_{\text {sol }}(L)\right]$.

(b) If $R_{\pi(k)}[E] \neq R_{\pi(k)}\left[E_{\text {sol }}(L)\right]$, then $R_{\pi(j)}[E] \neq R_{\pi(j)}\left[E_{\text {sol }}(L)\right]$, for some $j, 1 \leq j<k$.

Property (a) implies that at least one process can distinguish between an execution $E$ and an $L$-solo execution $E^{\prime}$ if the orders $\prec_{E}$ and $\prec_{E}^{\prime}$ differ. Property (b) implies that if the last process in the permutation $L$ distinguishes between $E$ and $E^{\prime}$, then some earlier process already distinguishes between them.

The intuition is the following: In an $L$-solo execution $E$, where $L=\left(p_{1}, \ldots, p_{k}\right)$, we have $p_{i} \prec_{E} p_{i+1}$, for every $i, 1 \leq i<k$. Moreover, if $j>i$, then process $p_{i}$ does not "know" whether or not process $p_{j}$ participates in $E$. Now consider another execution $E^{\prime}$, in which we "speed up" process $p_{k}$ slightly, so that it executes its orderoperation just before $p_{k-1}$ does but is still "unnoticed" by processes $p_{1}, \ldots, p_{k-2}$. Properties (a) and (b) imply that the records of both $p_{k-1}$ and $p_{k}$ are different in $E$ and $E^{\prime}$. Our proof uses this property to capture the required communication between $p_{k-1}$ and $p_{k}$.

Our results hold for algorithms that satisfy the following weak progress requirement.

DEFINITION 2. An implementation $\left(A_{1}, \ldots, A_{n}\right)$ satisfies weak obstruction-freedom if for every process $p_{i}$ and every execution $E$ after which all processes other than $p_{i}$ are idle, if $p_{i}$ runs by itself executing algorithm $A_{i}$ after $E$ then it eventually terminates.

Weak obstruction-freedom may be satisfied by an implementation regardless of whether or not it uses locks. It is easily seen that both deadlock-freedom and obstruction-freedom [11] imply weak obstruction-freedom. In what follows, we consider algorithms that satisfy weak obstruction-freedom. The rest of this section is devoted to proving the following theorem.

THEOREM 1. Let $\left(A_{1}, \ldots, A_{n}\right)$ be order encoding algorithms that satisfy weak obstruction-freedom. Then there is an execution $E \in \mathcal{E}$ with $\Omega(n \log n) R M R$ cost.

In the following we define the concept of critical operations. The number of critical operations performed during an execution is a lower bound on the number of RMRs incurred during that execution. Our proof technique encodes executions such that the code length is proportional to the number of critical operations.
DEFINITION 3. Consider an operation $\sigma$ of process $p$ on register $R$, that occurs right after some execution prefix $E$. If $\sigma$ is a readoperation, then it is critical if $p$ has not accessed $R$ in $E$, or $p$ has accessed $R$ in $E$, and some other process has written $R$ since p's last access of $R$. If $\sigma$ is a write-operation, then it is critical if $p$ has not written $R$ in $E$, or if p has written $R$ in $E$ and some other process has written $R$ since p's last write to $R$.

A similar notion of critical events was used by Anderson and Kim [2]; all operations that are critical according to our definition are also critical according to their definition (but not vice versa). A critical operation incurs an RMR in both write-through and write-back CC systems [2, pp. 226-227].

The general structure of our proof is the following. We construct $n$ ! different executions, each corresponding to a permutation $\pi \in S_{n}$ and denoted $E_{\pi}$. Our construction maintains the property that each process $p_{\pi(i)}$ does not "know" whether any processes $p_{\pi(j)}$, for $j>i$, participate in $E_{\pi}$. In that case, it is easily seen (as we prove later) that process $p_{\pi(1)}$ has to act exactly as it does in $E_{s o l}\left(L_{\pi}\right)$ and must also produce the same record. By an inductive argument, we show that all processes must produce the same record in $E_{\pi}$ and in $E_{s o l}\left(L_{\pi}\right)$. Indeed, assume that processes $p_{\pi(1)}, \ldots, p_{\pi(i)}$ produce the same record in both executions. Process $p_{\pi(i+1)}$ does not "know" whether any process other than $p_{\pi(1)}, \ldots, p_{\pi(i)}, p_{\pi(i+1)}$ participates in $E_{\pi}$. Hence if it records different values in $E_{\pi}$ and in $E_{\text {sol }}\left(L_{\pi}\right)$ it might violate property (b) of Definition 1. It follows that the record-vectors of $E_{\pi}$ and $E_{s o l}\left(L_{\pi}\right)$ are equal.

Since for each permutation $\pi$ the record vectors of $E_{\pi}$ and $E_{\text {sol }}\left(L_{\pi}\right)$ are equal, it follows that all executions $E_{\pi}$ are different. We then show that we can uniquely encode each execution $E_{\pi}$ and that the bit-length of each such encoding is bounded from above (up to a constant factor) by the total number of critical operations that are executed during $E_{\pi}$. Hence, the $n$ ! different executions $E_{\pi}$ lead to $n$ ! different codes. Therefore, the total number of critical operations in at least one of these executions is asymptotically at least the logarithm of the number of different records produced by these executions, i.e., $\Omega(\log (n !))=\Omega(n \log n)$. Below, we describe in detail the structure of the executions $E_{\pi}$.

\subsection{Codes and their Interpretation}

In this section, we define the structure of the executions $E_{\pi}$, and we define commands, such that a sequence of commands can be interpreted as an execution.

Consider an execution $E$. We say that a process $p$ loops locally after $E$, if $p$ does not terminate nor does it execute any critical operations in a solo-run that starts after $E$.

\subsubsection{Rounds and Sub-Rounds.}

In any execution $E_{\pi}$, processes proceed in rounds. Each round is composed of a sequence of 4 sub-rounds: read, early-write, latewrite, and non-critical sub-rounds. The very first sub-round is a read sub-round, followed by an early-write, then a late-write, and finally, a non-critical sub-round. After a round completes, the next round (if any) starts with another read sub-round, and so on.

The idea is that critical operations occur in one of the first three sub-rounds. If the critical operation is a read, then it occurs in the read sub-round, and if it is a write, it occurs in one of the write sub-rounds. The two different types of write sub-rounds allows to control which of the writes may become "visible" in the sense that they are not overwritten before some other process may read what was written. The executions we construct guarantee that whenever a process writes to a register in the early-write round, then some other process writes to the same register in the late-write round. Hence, writes in the early-write rounds are always "invisible". 
Each process may or may not wait during a round. If it waits, then it does not execute any steps in any of the four sub-rounds of the round. If it does not wait, it may execute at most one single step during the read, early-write, and late-write sub-rounds altogether, and then it may execute additional steps during the non-critical subround.

Now consider a round, and let $P \subseteq \mathcal{P}$ be the set of processes participating in that round. For process $p \in P$, let $\sigma_{p}$ be the operation $p$ is about to perform. Formally, $\sigma_{p}$ is a pair (op,reg), where $o p$ is either "read" or "write", and reg identifies the register on which this operation operates. Let $\lambda_{p} \in\{$ "critical" "non-critical" $\}$ denote whether $\sigma_{p}$ would be a critical operation, if it were executed as the first operation of the next round. Note that although $\lambda_{p}$ may be "noncritical", $\sigma_{p}$ may be critical when $p$ actually executes it, because by that time some other process may have already accessed the register which $\sigma_{p}$ accesses (our construction guarantees that this does not occur). On the other hand, from Definition 3 , if $\lambda_{p}=$ "critical" then $\sigma_{p}$ is guaranteed to be a critical operation when $p$ performs it.

Let $P^{\prime}=\left\{p \in P \mid \lambda_{p}=\right.$ "critical" $\}$. During the first three subrounds of a round (namely, the read, early-write, and late-write subrounds) each of the processes in $P^{\prime}$ performs its operation $\sigma_{p}$. No other operations are performed during these three sub-rounds. Those processes $p \in P^{\prime}$ for which $\sigma_{p}$ is a read-operation execute that operation in the read sub-round. We let these processes perform their read steps in increasing order of process IDs. After the read sub-round, those processes $p \in P^{\prime}$ for which $\sigma_{p}$ is a write-operation perform their writes. Some of these writes will be designated as early writes, and these will be performed in the early-write sub-round in increasing process ID order. The remaining writes are late writes and will be performed in the late-write sub-round, in increasing process ID order. As we prove later, the early writes performed in each round are overwritten by the late writes of that round.

The final sub-round is the non-critical sub-round, in which we schedule each of the remaining processes of $P^{\prime}$ in increasing order of their ID. We let each such process $p$ perform steps in the noncritical sub-round only if it is not about to loop locally. ${ }^{1}$ In this case, we let $p$ run solo until it is either about to perform a critical operation or until it terminates.

Order-operations play a special role by forcing processes to deviate from the round-scheme described above. A process $p$ that executes its order-operation continues to run solo until it terminates. We prove that, in the executions we consider below, $p$ cannot loop locally in such a solo run.

\subsubsection{Commands}

We associate a sequence $s_{i}$ of commands with each process $p_{i}$ such that a list $s_{1}, \ldots, s_{n}$ of command sequences determines an execution $E_{\pi}$. Each command is associated with a specific round of $E_{\pi}$, in which it becomes effective. It may then remain effective during some of the following rounds, until it ceases to be effective after some round.

Two types of commands exist: Wait commands and Participate commands. A Participate command indicates that the process will participate in the round in which the command becomes effective. The Participate command receives a single argument. If the process' first operation in that round is a critical write, then the argument indicates whether the write step is scheduled in the early-write or in

\footnotetext{
${ }^{1}$ If the algorithms are finite-state, then it can be determined by simulation whether a process will loop locally or not. We do not assume that, however. Thus, if the algorithms are infinite-state, then our proof is existential rather than constructive. In this case our proof assumes the existence of an oracle that determines whether or not a process will loop locally starting from a certain point.
}

the late-write sub-round. If the first operation of the process is not a critical write, then the argument is ignored. Thus, the Participatecommands are (1) Participate(late), and (2) Participate(early).

A Wait command indicates that the process should wait in the round in which the command becomes effective. The process may have to wait also in some of the following rounds. Thus, once the Wait command becomes effective, it may remain effective in a few additional rounds.

We now describe the Wait commands in detail and explain how we determine how long they remain effective. Consider a Wait command of process $p$ that becomes effective in round $i$. Let $R$ be the register that is about to be accessed by $p$ 's next operation.

1. Wait(next late-write): This command remains effective until (and including) the first round $j \geq i$, such that in round $j+1$ some process (other than $p$ ) will write to $R$ in the late-write sub-round.

2. Wait(reader/writer termination): This command remains effective until (and including) the first round $j>i$, in which one of the processes that access (read or write) $R$ during a round $i^{\prime} \in\{i+1, \ldots, j\}$ terminates.

Roughly speaking, Wait commands are used as follows. The first type of Wait commands is used for "hiding" a critical write by process $p$. If process $p$ is about to perform a critical write to register $R$ and this register is later written by an "earlier" process $q$ (i.e., $q \prec p$ ) whose write is not "hidden", then a Wait(next-write) command will make sure that $p$ 's write will be scheduled as an early write in the same round in which $q$ writes to $R$. Thus $p$ 's early write will be overwritten by $q$ 's late write.

Hiding critical writes is not always possible and the second type of Wait commands is used in such situations. If process $p$ is about to write to a register that is read later in the execution by an "earlier" process $q$, and if $p$ 's write cannot be "hidden", then the Wait(reader/writer termination) command is used to make sure that $p$ waits until $q$ terminates before it is allowed to perform its critical write. This way we can eliminate information flow from $p$ to "earlier" processes. Another situation, where we use this second type of Wait command is the following: If $R$ is local to process $q$ at the time when $p$ writes to $R$, then $p$ 's write operation changes the locality of $R$. Hence, $q$ 's next write operation on $R$ would now become a critical operation. The way we encode the commands, we have to be sure that the "criticality" of $q$ 's operation is not affected by "later" processes. We ensure this, by letting $p$ wait until $q$ 's execution is finished.

\subsubsection{The Interpretation of Commands}

We now describe how command sequences uniquely specify executions. An execution $E=C\left(s_{1}, \ldots, s_{n}\right)$, described by the command sequences $s_{1}, \ldots, s_{n}$, is determined as follows.

Assume for some $z \geq 1$, that the first $z-1$ rounds of execution $E$ have been determined and let $E^{\prime}$ denote the execution consisting of the first $z-1$ rounds (round 0 is defined to be the empty execution). Let $\sigma_{p}$ denote $p$ 's next step after $E^{\prime}$, i.e., $\sigma_{p}$ is a pair $(o p, r e g)$, where $o p$ indicates whether the step is a read- or write-operation, and reg identifies the register on which this operation operates. Further, let $\lambda_{p} \in\{$ critical, non-critical $\}$ indicate whether or not $\sigma_{p}$ is critical if it is scheduled immediately after $E^{\prime}$.

For each process $p$, we now determine which command (if any) will be effective in round $z$. The exact behavior of process $p$ is then uniquely determined by the structure of rounds described above and by the following rules:

1. If a Wait command is effective in round $z$, then process $p$ does not take any steps in this round. 
2. If a Participate $(x)$ command ( $x \in\{$ early, late $\}$ ) is effective in round $z$, then process $p$ executes $\sigma_{p}$ in the read sub-round if it is a read-operation, and otherwise it executes $\sigma_{p}$ in the $x$-write sub-round. It also executes non-critical steps in the non-critical sub-round (as described in Section 3.2.1), unless it is about to loop locally.

3. If no command is effective in round $z$, then process $p$ executes only non-critical steps in the non-critical sub-round (as described in Section 3.2.1), unless it is about to loop locally.

Hence, to establish the uniqueness of the execution specification, it suffices to show how to determine which command (if any) is effective in each round. Let $c_{p}^{\prime}$ denote the last command of $p$ that became effective in $E^{\prime}$ (if such a command exists), and let $c_{p}$ denote the command following $c_{p}^{\prime}$ in $p$ 's sequence of commands (if it exists). If $c_{p}^{\prime}$ and $c_{p}$ do not exist, then no command is effective in round $z$.

In round $z$, command $c_{p}^{\prime}$ may either remain effective or it may cease to be effective before round $z$ starts. Even if $c_{p}^{\prime}$ ceases to be effective (or does not exist), $c_{p}$ might not become effective in round $z$. In fact, $c_{p}$ becomes effective in round $z$ if and only if $c_{p}^{\prime}$ ceased to be effective before the start of round $z$ and if $\lambda_{p}=$ critical.

It remains to describe how to decide whether $c_{p}^{\prime}$ ceases to be effective right after round $z-1$, or whether it remains effective during round $z$. If $c_{p}^{\prime}$ is a Wait(reader/writer termination) command that was effective in round $z-1$ then it is uniquely determined by the previous rounds whether it remains effective in round $z$ (see the description of the Wait commands in Section 3.2.2). It remains to consider the case where $c_{p}^{\prime}=$ Wait(next late-write).

Let $P^{\prime}$ denote the set of processes $p$ for which $c_{p}^{\prime}=$ Wait(next latewrite) and $c_{p}^{\prime}$ was effective in round $z-1$. For all processes not in $P^{\prime}$ it is already known, which command is effective in round $z$, so we know whether a process $q \in P-P^{\prime}$ writes in the late-write sub-round of round $z$. Thus, we can apply the following rule:

For $p \in P^{\prime}$ and $\sigma_{p}=(o p, R)$, command $c_{p}^{\prime}$ ceases to be effective right after round $z-1$, if and only if there is a process $q \in P-P^{\prime}$ that writes in the late-write sub-round of round $z$ to register $R$.

This rule would not be compatible with our commands definition, if there were a process $p^{\prime} \in P^{\prime}$ that would now write in the late-write sub-round of round $z$ to register $R$. But this cannot happen, as our encodings will guarantee that no process $p^{\prime} \in P^{\prime}$ will "write late" in round $z$. This is ensured by the following invariant:

Every Wait(next late-write) in the commands sequence of a process is immediately followed

by a Participate(early) command.

Hence, we know whether or not there is a process that "writes late" to $R$ in round $z$ before considering the processes of $P^{\prime}$.

The specifications of rounds and commands and their interpretation guarantees that every list $\left(s_{1}, \ldots, s_{n}\right)$ of command sequences uniquely determines an execution $C\left(s_{1}, \ldots, s_{n}\right)$. Note that this does not imply that all processes terminate in this execution.

\subsection{Execution Encoding}

In this section we show how to find for every permutation $\pi$ command sequences $s_{1}, \ldots, s_{n}$ defining an execution $E_{\pi}=C\left(s_{1}, \ldots, s_{n}\right)$ which has the same record vector as $E_{s o l}\left(L_{\pi}\right)$.
DEFINITION 4. Let $E$ and $E^{\prime}$ be two executions, and let $\sigma_{i}$ and $\sigma_{i}^{\prime}$, for $i \in \mathbb{N}$, be the $i$-th step a process $p$ makes during executions $E$ and $E^{\prime}$, respectively; If p makes fewer than i steps in $E\left(E^{\prime}\right)$, then $\sigma_{i}$ $\left(\sigma_{i}^{\prime}\right)$ is defined to be $\perp$. The executions $E$ and $E^{\prime}$ are indistinguishable for process $p$, denoted $E \sim_{p} E^{\prime}$, if the following statements hold for every $i \in \mathbb{N}$ :

1. $\sigma_{i}=\perp$ if and only if $\sigma_{i}^{\prime}=\perp$.

2. If $\sigma_{i}$ and $\sigma_{i}^{\prime}$ are read-operations, then both operations return the same response.

3. $\sigma_{i}$ is a critical operation if and only if $\sigma_{i}^{\prime}$ is a critical operation.

The first property ensures that both processes perform an equal number of steps in $E$ and $E^{\prime}$. The second and third properties ensure that $p$ is in the same state after performing $i$ steps in $E$ and in $E^{\prime}$. If $p$ is in the same state after $i$ steps in $E$ and $E^{\prime}$, then, clearly, the type of operation in the $(i+1)$ 'th step (i.e., read or a write) and the register on which it operates are the same in $E$ and $E^{\prime}$. Hence, $\sigma_{i+1}=\sigma_{i+1}^{\prime}$.

For the rest of this section we fix a permutation $\pi$. Every process $p_{i}$ executes algorithm $A_{i}$, and the sequence $\left(A_{1}, \ldots, A_{n}\right)$ of algorithms is order encoding and satisfies weak obstruction-freedom. We encode processes' steps in the order prescribed by $\pi$. That is, first we construct the command sequence $s_{\pi(1)}$ for $p_{\pi(1)}$, then the command sequence $s_{\pi(2)}$ for $p_{\pi(2)}$, and so on. The sequences $s_{\pi(1)}, \ldots, s_{\pi(k)}$ determine an execution $C\left(s_{1} \ldots s_{n}\right)$, where $s_{\pi}(j)$ is the empty command sequence for $j>k$. Thus, the processes $p_{\pi(k+1)}, \ldots, p_{\pi(n)}$ do not take any steps in $C\left(s_{1} \ldots s_{n}\right)$ (recall that the first operation of a process is always critical, and if a process' command sequence is empty, then it can only participate in the non-critical sub-rounds where it can't execute a critical operation).

In order to simplify notation, we let $q_{i}=p_{\pi(i)}$ and $a_{i}=s_{\pi(i)}$ for $1 \leq i \leq n$. We also let $D\left(a_{1}, \ldots, a_{k}\right)$ denote the execution $C\left(s_{1}, \ldots, s_{n}\right)$, where for all $j>k, s_{\pi(j)}$ is the empty command sequence.

\subsubsection{Invariants}

Our encoding needs to ensure that Invariant (1), defined in Section 3.2.3, holds. In addition, our encoding will maintain (as we prove) the following invariants. Assume we have constructed $s_{1}, \ldots, s_{k}$, then

$$
\begin{aligned}
& D\left(a_{1}, \ldots, a_{i}\right) \sim{ }_{q_{i}} D\left(a_{1}, \ldots, a_{j}\right) \\
& \text { for every } i, j, 1 \leq i \leq j \leq k, \text { and }
\end{aligned}
$$

$$
\text { in } D\left(a_{1}, \ldots, a_{k}\right) \text {, all processes } q_{1}, \ldots, q_{k} \text { terminate. }
$$

When $k=n$ we have

$$
D\left(a_{1}, \ldots, a_{i}\right) \sim_{q_{i}} D\left(a_{1}, \ldots, a_{n}\right) \quad\left(=C\left(s_{1}, \ldots, s_{n}\right)\right),
$$

for every $i, 1 \leq i \leq n$.

Proposition 2. Let $E_{\pi}^{k}=D\left(a_{1}, \ldots, a_{k}\right)$ and $L_{\pi}^{k}=\left(q_{1}, \ldots, q_{k}\right)$. If Invariants (2) and (3) hold then

(a) $\vec{R}\left[E_{\pi}^{k}\right]=\vec{R}\left[E_{\text {sol }}\left(L_{\pi}^{k}\right)\right]$.

(b) Each process $q_{i}, 1 \leq i<k$, terminates before process $q_{i+1}$ executes its order operation.

Proof. (a) Let $\left(\alpha_{1}, \ldots, \alpha_{k}\right)=\vec{R}\left[E_{\pi}^{k}\right]$ and $\left(\beta_{1}, \ldots, \beta_{k}\right)=$ $\vec{R}\left[E_{\text {sol }}\left(L_{\pi}^{k}\right)\right]$. From Invariant (2), executions $E_{\pi}^{k}$ and $D\left(a_{1}\right)$ are indistinguishable to process $q_{1}$. They are thus also indistinguishable 
to $q_{1}$ from a solo-execution. Since $q_{1}$ terminates in $E_{\pi}^{k}$ by Invariant (3), it has to record $\beta_{1}$. Thus, $\alpha_{1}=\beta_{1}$.

Now assume that $\left(\alpha_{1}, \ldots, \alpha_{i}\right)=\left(\beta_{1}, \ldots, \beta_{i}\right)$ for $1 \leq i \leq k$. We prove that $\alpha_{i+1}=\beta_{i+1}$. First note that, by Invariant (2), $\vec{R}\left[D\left(a_{1}, \ldots, a_{i+1}\right)\right]=\left(\alpha_{1}, \ldots, \alpha_{i+1}\right)$. Moreover, from definitions, $\vec{R}\left[E_{\text {sol }}\left(L_{\pi}^{i+1}\right)\right]=\left(\beta_{1}, \ldots, \beta_{i+1}\right)$, where $L_{\pi}^{i+1}=\left(q_{1}, \ldots, q_{i+1}\right)$. Thus, by Definition 1 (b), if $\alpha_{i+1} \neq \beta_{i+1}$ then $\left(\alpha_{1}, \ldots, \alpha_{i}\right) \neq\left(\beta_{1}, \ldots, \beta_{i}\right)$, which is a contradiction.

(b) We prove the claim by induction on $i$. For $i=0$ the claim holds vacuously. Assume $i \geq 1$. Consider the point in execution $E_{\pi}^{k}$ when $q_{i}$ executes its order operation. By induction hypothesis, processes $q_{1}, \ldots, q_{i-1}$ have already terminated. By definition of the round scheme, $q_{i}$ runs solo starting from this point until it terminates, if it ever does. Since algorithms $A_{i}$ satisfy weak obstruction-freedom, $q_{i}$ eventually terminates when it runs solo after its order operation in $D\left(a_{1}, \ldots, a_{i}\right)$. By Invariant (2), $q_{i}$ cannot distinguish $E_{\pi}^{k}$ from $D\left(a_{1}, \ldots, a_{i}\right)$. It follows that $q_{i}$ eventually terminates when it runs solo after its order operation also in $E_{\pi}^{k}$. Finally note that $q_{i+1}$ could not have executed its order-operation before $q_{i}$ : assuming otherwise implies $q_{i+1} \prec_{E} q_{i}$, and, by Definition 1 (a), the records of $\vec{R}\left[E_{\pi}^{k}\right]$ and $\vec{R}\left[E_{\text {sol }}\left(L_{\pi}^{k}\right)\right]$ must differ. This would contradict part (a).

Part (a) of Proposition 2 establishes that every process $p_{i}$ records the same values in executions $C\left(s_{1}, \ldots, s_{n}\right)$ and $E_{s o l}\left(L_{\pi}\right)$. Let $E_{\pi}=$ $C\left(s_{1}, \ldots, s_{n}\right)$, then the record-vector of $E_{\pi}$ can be encoded by a string of length $O\left(\sum_{i=1}^{n}\left|s_{i}\right|\right)$. Assuming the above invariants hold (as we prove in the following section), it follows from Proposition 2 and Definition 1 (a) that every execution $E_{\pi}$ yields a distinct recordvector.

\subsubsection{Realization of the Encoding}

We now describe how we construct the executions $E_{\pi}$ and encode them so as to maintain Invariants (1)-(3) for $k=1, \ldots, n$. For $k=1$, we consider a solo run of process $q_{1}$, and we let $t$ be the total number of critical operations it executes. We then let $a_{1}$ be the concatenation of $t$ Participate(late) commands. Thus, $D\left(a_{1}\right)$ is a complete solorun of process $q_{1}$, and during each round $q_{1}$ is performing exactly one critical operation (either a read in the read sub-round, or a latewrite in the late-write sub-round). Moreover, by weak obstructionfreedom and since $s_{1}$ contains no Wait commands, Invariants (1), (2) and (3) hold for $k=1$.

Now assume that $a_{1}, \ldots, a_{k-1}$, for $k \geq 2$, have been determined, so that Invariants (1), (2), and (3) hold. We construct the commands sequence $a_{k}=\left(c_{1}, \ldots, c_{\ell}\right)$ of process $q_{k}$. In order to satisfy (1), whenever we add a command Wait(next late-write), we add a command Participate(early) immediately after it. In this case, we abuse notation and let $c_{i}$ denote a sequence consisting of both these commands.

Assume that we have constructed $a_{k}^{t}:=\left(c_{1}, \ldots, c_{t}\right)$. We have to ensure the following two invariants.

$$
\begin{aligned}
& D\left(a_{1}, \ldots, a_{i}\right) \sim_{q_{i}} D\left(a_{1}, \ldots, \ldots, a_{k-1}, a_{k}^{t}\right) \\
& \text { for every } i, 1 \leq i<k, \text { and }
\end{aligned}
$$

in $D\left(a_{1}, \ldots, \ldots, a_{k-1}, a_{k}^{t}\right)$, all commands in $a_{k}^{t}$

first become effective and then cease to be effective.

Note that this implies that Invariant (2) is true if we let $a_{k}=a_{k}^{t}$, but Invariant (3) is not necessarily true since $q_{k}$ might not terminate during that execution. Certainly this is the case for $t=0$ (i.e., if $a_{k}^{t}$ is the empty sequence of commands) because in that case $D\left(a_{1}, \ldots, a_{k-1}, a_{k}^{0}\right)$ is the same execution as $D\left(a_{1}, \ldots, a_{k-1}\right)$ but $a_{k}$ takes no steps in this execution.

However, if we can continuously extent the command sequence $a_{k}^{t}$ such that (4) and (5) remain true, then we will eventually ob- tain a command sequence $a_{k}^{t^{*}}$, such that process $q_{k}$ terminates in $D\left(a_{1}, \ldots, a_{k-1}, a_{k}^{t^{*}}\right)$, too. This is because there exists an integer $T$ such that after $T$ rounds all processes $q_{1}, \ldots, q_{k-1}$ have terminated, and thus all commands in $a_{k}^{t^{*}}$ that become effective in a round later than $T$ must be Participate commands in order not to violate (5). By weak obstruction-freedom, process $q_{k}$ must eventually terminate.

So assume we have constructed $D^{t}=D\left(a_{1}, \ldots, a_{k-1}, a_{k}^{t}\right)$ for some $t \geq 0$, such that (4) and (5) hold. If process $q_{k}$ terminates in $D^{t}$, then we are done and we let $a_{k}=a_{k}^{t}$. Invariants (2) and (3) now hold.

Assume now that $q_{k}$ does not terminate in $D^{t}$. Invariant (4) ensures that processes $q_{1}, \ldots, q_{k-1}$ act in $D^{t}$ exactly as in $D\left(a_{1}, \ldots, a_{k-1}\right)$. Hence, they all terminate. Moreover, by Invariant (5), there exists some round $z-1$ of execution $D^{t}$ after which the last command of $a_{k}^{t}$ ceases to be effective.

Consider the following round $z$. Let $\sigma$ denote the operation that $q_{k}$ is about to perform, and let $\lambda=$ "critical" if and only if $\sigma$ is critical when executed as the first operation of round $z$. Let $R$ be the register accessed by $\sigma$. In the following we determine the next command $c_{t+1}$ to be appended to $q_{k}$ 's sequence of commands. I.e., $a_{k}^{t+1}$ is the concatenation of $a_{k}^{t}$ and $c_{t+1}$, and $D^{t+1}=D\left(a_{1}, \ldots, a_{k-1}, a_{k}^{t+1}\right)$. We then argue that

$$
\forall 1 \leq i<k: D^{t} \sim_{q_{i}} D^{t+1}
$$

This will imply Invariant (4) by transitivity of $\sim_{q_{i}}$. If $c_{t+1}$ is a Wait command, we will also argue that Invariant (5) remains true. Potentially, there are three ways in which appending a command $c_{t+1}$ to $q_{k}$ 's commands sequence may lead to a violation of Invariant (6):

1) $\sigma$ is a write to $R$ : then some other process $q_{i}, i<k$, may either read what $q_{k}$ writes, or it may notice a "change in locality" of $R$ (one of $q_{i}$ 's non-critical commands on $R$ may become critical due to $q_{k}$ 's write).

2) $\sigma$ is a write or read command and process $q_{k}$ terminates: then this may change the semantics of the command sequence of some process $q_{i}, i<k$. This may only happen if, during the round in which $q_{k}$ terminates, $q_{i}$ 's effective command is Wait(reader/writer termination), and $q_{i}$ is on the verge of writing to register $R$. In this case, $q_{i}$ 's Wait command may cease to be effective in execution $D^{t+1}$ before it ceases to be effective in $D^{t}$.

3) $c_{t+1}$ is a Participate command, and $q_{k}$ executes a non-critical write in the non-critical sub-round of round $z$, that will make the execution distinguishable for some process $q_{i}, i<k$. This cannot happen: If it does, then $q_{k}$ must have executed a critical write on $R$ in some earlier round $z^{\prime}<z$, and no other process has written to $R$ since then. It follows that $q_{k}$ 's write in round $z^{\prime}$ has already led to an execution that can be distinguished by process $q_{i}$ from $D\left(a_{1}, \ldots, a_{k-1}\right)$. This implies in turn that Invariant (4) was violated even before we added $c_{t+1}$ to $q_{k}$ 's commands list.

We now prove that Proposition 2 precludes problem 2).

Proposition 3. Let $D^{*}$ be the execution obtained from the command sequences $a_{1}, \ldots, a_{k-1}, a_{k}^{t+1}$ by stopping $q_{k}$ just before it is about to terminate (if it terminates). If $D^{t} \sim_{q_{i}} D^{*}$ for all $1 \leq i<k$, then $D^{t} \sim_{q_{i}} D^{t+1}$ for all $1 \leq i<k$.

ProOF. From the definition of $D^{*}$, it is not possible that $q_{k}$ 's termination in $D^{*}$ causes a Wait(reader/writer termination) command by process $q_{i}, i<k$, to become effective. If $q_{k}$ is not stopped in $D^{*}$, then $D^{*}=D^{t+1}$. Assume, then, that $q_{k}$ is stopped in $D^{*}$ just before it terminates. It is enough to show that $q_{k}$ terminates in $D^{*}$ only after $q_{1}, \ldots, q_{k-1}$ terminate. By Invariant (3), all processes $q_{i}$, $i<k$, terminate in $D^{t}$ and, since $D^{t} \sim_{i} D^{*}$, they terminate also in $D^{*}$. We are therefore under the conditions of Proposition 2. Applying Proposition 2, we get that processes $q_{1}, \ldots, q_{k-1}$ terminate in $D^{*}$ before process $q_{k}$ executes its order operation. 
We now describe how $c_{t+1}$ is determined. The following cases exist.

Case A: $\lambda=$ "critical". Then $\sigma$ will be a critical operation when it is executed. We handle read and write critical operations differently as follows.

Case A.1: $\sigma$ is a read operation. Then $c_{t+1}$ is set to be Participate(late). Thus, in $D^{t+1}, \sigma$ is added to the read sub-round of round $z$ and $q_{k}$ 's following non-critical operations are added to round $z$ 's non-critical sub-round. Clearly, $\sigma$ cannot be observed by other processes. Also, from Definition 3, $\sigma$ cannot change the criticality of other operations. Hence, Invariant (6) holds and therefore also Invariant (4). Since $c_{t+1}$ is not a Wait command, and since $q_{k}$ does not terminate in $D^{t}$, Invariant (5) holds vacuously.

Case A.2: $\sigma$ is a write operation. We consider several subcases, depending on execution $D^{t}$. In what follows, we let $q_{\text {late }}^{W}$ denote the first process that writes to $R$ in the late-write sub-round of a round $z_{\text {late }}^{W} \geq z$ in $D^{t}$. If no such process exists, we denote $z_{\text {late }}^{W}=\infty$ and say that $q_{\text {late }}^{W}$ is undefined.

Case A.2.1: $z_{\text {late }}^{W}=z$. Then we set $c_{t+1}$ to Participate(early). Consider execution $D^{t+1}$. In round $z$, process $q_{k}$ writes to register $R$ in the early-write sub-round. In the same round, $q_{k}$ 's write will be overwritten by process $q_{\text {late }}^{W}$ that writes to $\mathrm{R}$ in the late-write sub-round. Since no reads occur in the early- or late-write sub-rounds, no process ever reads the value written by $q_{k}$ to $\mathrm{R}$. Moreover, all writes that occur in the early- and late-write sub-rounds are critical. Thus, after the late-write of process $q_{\text {late }}^{W}$ to register $R, R$ is local to $q_{\text {late }}^{W}$ regardless of the write by $q_{k}$. Thus Invariant (6) holds, and therefore also Invariant (4). Finally, since $c_{t+1}$ is not a Wait command, Invariant (5) holds vacuously.

Case A.2.2: $z<z_{\text {late }}^{W}<\infty$. Then $c_{t+1}$ is a sequence of two commands: a Wait(next late-write) command followed by a Participate(early) command. Thus, process $q_{k}$ waits in $D^{t+1}$ until some process writes to register $R$ in the late-write sub-round of some round $z^{\prime} \geq z$. The Participate(early) command becomes effective in round $z^{\prime}$. Process $q_{\text {late }}^{W}$ writes in execution $D^{t}$ to register $R$ in round $z_{\text {late }}^{W}>z$. Clearly, as long as $q_{k}$ 's Wait(next late-write) command remains effective, the execution is indistinguishable from $D^{t}$ to all processes, so process $q_{\text {late }}^{W}$ writes to $R$ in round $z_{\text {late }}^{W}$ also in $D^{t+1}$. It follows that $z^{\prime}=z_{\text {late }}^{W}$. Thus, after sub-round $z^{\prime}-1, q_{k}$ 's Wait(next late-write) command ceases to be effective and its Participate(early) becomes effective. Clearly, Invariant (5) holds in this case. Moreover, after round $z^{\prime}-1$ we are under the conditions of Case A.2.1. and so Invariant (4) also holds.

Case A.2.3: $z_{\text {late }}^{W}=\infty$.

A.2.3 (a): If none of the processes in $\left\{q_{1}, \ldots, q_{k-1}\right\}$ access register $R$ in a round $z^{\prime} \geq z$, then we let $c_{t+1}$ be Participate(late). Invariants (4) and (5) clearly hold in this case.

A.2.3 (b): Assume that process $q_{j}, 1 \leq j<k$, accesses register $R$ during round $z^{\prime} \geq z$. In this case, we must not allow process $q_{k}$ to write register $R$ since we cannot "hide" this write. Therefore, $q_{k}$ has to wait until the next process that will read $R$ terminated. We set $c_{t+1}$ to be Wait(reader/waiter termination). Since this is a Wait command, Invariant (4) holds. Additionally, it is clear that the Wait command ceases to be effective after the sub-round in which $q_{j}$ terminates, or even before that. Hence Invariant (5) holds.

Case B: $\lambda=$ "non-critical". From our construction, the next command does not become effective in round $z$. Instead, process $q_{k}$ will simply participate in the following non-critical sub-round. Therefore no command is encoded for this round. Instead, we consider the first round $z^{\prime}>z$ at which $q_{k}$ 's next command will become effective. Such a round must exist, since by (3) all processes $q_{1}, \ldots, q_{k-1}$ terminate in $D^{t}$, and once they have all terminated, $q_{k}$ cannot loop locally.

\subsubsection{Length of the Encoding}

We now bound the the number of commands in the command sequences obtained above in terms of the number of critical operations that occur in $E_{\pi}:=D\left(a_{1}, \ldots, a_{n}\right)$.

LEMMA 4. The total number of critical operations in execution $D=D\left(a_{1}, \ldots, a_{n}\right)$ is $\Omega\left(\sum_{i=1}^{n}\left|a_{i}\right|\right)$, where $\left|a_{i}\right|$ is the length of command sequence $a_{i}$.

We need the following simple observations.

OBSERVATION 5. In the sequence $a_{k}$ of commands, a Wait(reader/writer termination) command is always followed directly either by another Wait(reader/writer termination) command, or by a Participate(late) command.

PROOF. By construction (Case A.2.3 (b)) after a round in which a Wait(reader/writer termination) command $c$ becomes effective for process $q_{k}$, none of the processes in $\left\{q_{1}, \ldots, q_{k-1}\right\}$ performs a latewrite on register $R$. (I.e., $z_{\text {late }}^{W}=\infty$.) This situation cannot change until the Wait command $c$ ceases to be effective (and not even after that). Hence, the command that follows $c$ must be either another Wait(reader/writer termination) command or a Participate(late) command. Clearly, $c$ is followed by another command, because $q_{k}$ terminates in $D\left(a_{1}, \ldots, a_{k}\right)$ (Invariant (3)).

OBSERVATION 6. If $k$ processes access the same register $R$ during an execution, then the total number of critical operations incurred on register $R$ is at least $k-1$.

PROOF. It follows right away from Definition 3, that after the first process has accessed register $R$, the first operation of each other process on register $R$ is a critical operation.

The remainder of this section is devoted to the proof of Lemma 4. First of all note that every command in the command sequences takes effect in some round. (This is implicit in the construction, but even if it weren't, we could simply remove every command that does not take effect without changing the execution.) By definition of the round-scheme, process $q_{i}$ executes at least one critical operation for each Participate command in $a_{i}$. Moreover, by Invariant (1), the number of Wait(next late-write) commands in $a_{i}$ is bounded by the number of Participate (early) commands in $a_{i}$. Hence, it suffices to show that the total number of Wait(reader/writer termination) commands in $a_{1}, \ldots, a_{n}$ is bounded up to a constant factor by the number of critical operations.

We can associate each Wait(reader/writer termination) command with a unique pair $(b, z)$, where $b$ is the process in whose command sequence this command appears, and $z$ is the round at which the command takes effect in execution $D$.

Let $\mathcal{W}$ be the set of all pairs $(b, z)$ associated with a Wait(reader/writer termination) command. Further, let $\mathcal{R}$ be the set of all registers, and recall that $\mathcal{P}=\left\{p_{1}, \ldots, p_{n}\right\}$ is the set of processes, and that $q_{i}=p_{\pi(i)}$.

Claim 7. There exist two mappings $f: \mathcal{W} \rightarrow \mathcal{P}$ and $g: \mathcal{W} \rightarrow$ $\mathcal{R}$ such that for all $(b, z) \in \mathcal{W}$ it holds

(a) $f(b, z) \neq b$, and

(b) in execution $D$, process $b$ writes register $g(b)$ and process $f(b)$ accesses register $g(b)$, and

(c) for all $R \in \mathcal{R}$, function $f$ is injective on $g^{-1}(R)$. 
Proof. Consider a pair $(b, z) \in \mathcal{W}$, where $b=q_{k}$. By construction (see Case A.2.3 above), in round $z$ of execution $D$ process $q_{k}$ is on the verge of critically writing to a register $R$, and none of the processes in $\left\{q_{1}, \ldots, q_{k-1}\right\}$ writes to register $R$ in a late-write subround of a round $z^{\prime}>z$. Also by the assumption of that case, there exists a process in $\left\{q_{1}, \ldots, q_{k-1}\right\}$ that accesses register $R$ in a round $z^{\prime}>z$. Among those, let $q_{j}$ be the process that terminates first. We define $f(b, z):=q_{j}$ and $g(b, z):=R$. Obviously (a) and (b) are true because $q_{j} \neq b$ (by construction, $b$ waits until $q_{j}$ terminates), $q_{j}$ accesses register $R$ and $b$ writes to register $R$.

We now show that $f$ is injective on $W_{R}=g^{-1}(R)$. For the purpose of a contradiction assume that there exist two pairs $\left(q_{i}, z_{i}\right)$ and $\left(q_{j}, z_{j}\right)$ and a process $q_{k} \in \mathcal{P}$, such that $g\left(q_{i}, z_{i}\right)=g\left(q_{j}, z_{j}\right)=$ $R$ and $f\left(q_{i}, z_{i}\right)=f\left(q_{j}, z_{j}\right)=q_{k}$. Let $w_{i}$ and $w_{j}$ be the two Wait(reader/writer termination) commands associated with the two pairs.

First assume that $i=j$, w.l.o.g. $z_{j}>z_{i}$. Hence, process $q_{i}$ 's Wait command $w_{i}$ ceases to be effective before the sub-round in which its Wait command $w_{j}$ takes effect. But by definition of $f, w_{i}$ ceases to be effective right after the round in which process $q_{k}$ terminates. Hence, when $w_{j}$ becomes effective, process $q_{k}$ has already terminated, so $f\left(q_{j}, z_{j}\right)$ cannot be that process-a contradiction.

Now assume that $i \neq j$, w.l.o.g. $i<j$. Then the assumption $f\left(q_{i}\right)=f\left(q_{j}\right)=q_{k}$ implies that $q_{k}$ terminates in a round $z^{\prime}$, where $z^{\prime}>z_{i}$ and $z^{\prime}>z_{j}$, and the Wait commands $w_{i}$ and $w_{j}$ remain effective until then. By Observation 5 the commands $w_{i}$ and $w_{j}$ are both followed by a (possibly empty) sequence of additional Wait(reader/writer-termination) commands, and eventually one Participate(late) command. Hence, there exists a round $z_{i}^{*} \geq z^{\prime}>$ $\max \left\{z_{j}, z_{i}\right\}$ in which process $q_{i}$ is writing to register $R$ in a late-write sub-round.

Now consider the situation when we added command $w_{j}$ to the command sequence of process $q_{j}$. Let $a_{j}^{t}$ be the commands that appear in that command sequence before $w_{j}$. In execution $D\left(a_{1}, \ldots, a_{j-1}, a_{j}^{t}\right)$, process $q_{i}$ is acting exactly in the same way as in execution $D$ (because of indistinguishability). When we added the Wait(reader/writer termination) command $w_{j}$ to the sequence $a_{j}^{t}$, then we must have been in Case A.2.3 (b) for $z=z_{j}$. But the fact (recall $i<j$ ) that $q_{i}$ is writing in execution $D\left(a_{1}, \ldots, a_{j-1}, a_{j}^{t}\right)$ in round $z_{i}^{*}>z_{j}$ to register $R$ in a late-write sub-round, means that we are actually in a case where $z_{\text {late }}^{W} \leq z_{i}^{*}<\infty$. This contradicts the assumption of Case A.2.3 (b).

We argue that the claim implies that the number of critical operations during $D$ is $\Omega(|\mathcal{W}|)$. Let $W_{R}=g^{-1}(R)$ for $R \in \mathcal{R}$. Since $\mathcal{W}$ is the disjoint union of all $W_{R}, R \in \mathcal{R}$, it suffices to show that the total number of critical operations on register $R$ is $\Omega\left(\left|W_{R}\right|\right)$. Assume that $\left|W_{R}\right|>0$ and let $Q=f\left(W_{R}\right)$. By (b), all processes in $Q \cup W_{R}$ access register $R$ during execution $D$. Hence, by Observation 6 the total number of critical operations on register $R$ is at least $\left|Q \cup W_{R}\right|-1$. By (a), $\left|Q \cup W_{R}\right| \geq 2$, and by injectivity of $f$ on $W_{R}$ (c), we have $|Q|=\left|W_{R}\right|$. Hence, the total number of critical operations on register $R$ is at least

$$
\left|Q \cup W_{R}\right|-1 \geq \max \left\{2,\left|W_{R}\right|-1\right\}=\Omega\left(\left|W_{R}\right|\right) .
$$

PRoOF of THEOREM 1 . Let $E_{\pi}$ be the execution $D\left(a_{1}, \ldots, a_{n}\right)$ we have constructed for permutation $\pi$. By Proposition 2, the record-vector of $E_{\pi}$ is the same as that of $E_{s o l}\left(L_{\pi}\right)$. By Definition 1 , the record-vectors $\vec{R}\left[E_{\text {sol }}\left(L_{\pi}\right)\right]$ are different for all permutations $\pi$. Hence, the record-vectors $\vec{R}\left[E_{\pi}\right]$, for $\pi \in S_{n}$, are all different. Since there are $n$ ! permutations, there exists a permutation $\pi$, such that the encoding of $E_{\pi}=D\left(a_{1}, \ldots, a_{n}\right)=C\left(s_{1}, \ldots, s_{n}\right)$ uses at least $\log (n !)=\Omega(n \log n)$ bits. Since each encoding of a permutation consists of $n$ sequences $s_{1}, \ldots, s_{n}$ of commands, and for each command there are only four possibilities, it follows that $\left|s_{1}\right|+\cdots+\left|s_{n}\right|=\left|a_{1}\right|+\cdots+\left|a_{n}\right|=\Omega(n \log n)$. By Lemma 4, the number of critical operations executed in $E_{\pi}$ is $\Omega(n \log n)$.

\section{LOWER BOUND IN THE DSM MODEL}

In this section we provide a high-level description of how the proof of Theorem 1, presented in Section 3 for the CC model, can be adapted in a simple manner to obtain an equivalent result for the Distributed Shared Memory (DSM) model. The complete proofs are deferred to the full paper.

Recall that the proof of Theorem 1 for the $\mathrm{CC}$ model uses the concept of critical operations. While the number of CC critical operations (as given in definition 3) is, in general, smaller than the number of RMRs, for the DSM model we define each RMR as a critical operation.

DEFINITION 5. An operation $\sigma$ of process $p$ on register $R$ is critical in a DSM system, if $R$ is remote to p's processor.

The general structure of the DSM proof is identical to that of the CC proof. A set of executions $\left\{E_{\pi} \mid \pi \in S_{n}\right\}$ is constructed and maintains the same properties maintained by the CC executions. The key change is in the set of commands used for execution encoding. Recall that two types of Wait commands are used for encoding CC executions: Wait(next late-write) and Wait(reader/writer termination). A third type of Wait commands is used for DSM encodings, motivated by the need to prevent information flow from "later" processes to "earlier" processes. Fix a permutation $\pi \in S_{n}$ and consider the steps taken by $q_{j}$, the $j$ 'th process by $\pi$. Assume that a set of earlier processes, $Q \in\left\{q_{1}, \ldots, q_{j-1}\right\}$, each access $q_{j}$ 's local registers. For $E_{\pi}$ to possess the required properties, we schedule $q_{j}$ 's steps so that no process in $Q$ reads a value written by $q_{j}$. Specifically, we must prevent processes in $Q$ from reading local writes made by $q_{j}$. Unlike in the CC proof, here we cannot enforce this by encoding $q_{j}$ 's accesses of local registers since then the encoding length may asymptotically exceed the number of critical steps. Instead, we delay scheduling of $q_{j}$ 's steps until after all the processes in $Q$ terminate. We use a new Wait(early-process-termination) command to enforce this. The code of process $q_{j}$ therefore begins with $k$ such wait commands, where $k=|Q|^{2}$

Another DSM scheduling requirement (symmetric, in a sense, to the previous one) is that we must not allow a process to communicate information to earlier processes via their local segments. If the next access by $q_{j}$ is a write to a register $R$ local to $q_{i}$, for some $i<j$, and if $q_{i}$ accesses $R$ in its execution, then $q_{i}$ must terminate before $q_{j}$ is allowed to proceed. This requirement is already met, however, by the encoding and execution construction for the $\mathrm{CC}$ model, since in such a case a Wait(reader/writer termination) command is appended to $q_{j}$ 's code on account of $q_{i}$ 's access of $R$. (See Case A.2.3 (b) in Section 3.3.2.)

The proofs of invariants 1-6 are similar to those for the CC model; they are actually somewhat simpler, since the locality of registers is fixed. The following lemma is the DSM equivalent of Lemma 4.

LEMMA 8. The total number of critical operations in a DSM execution $D=D\left(a_{1}, \ldots, a_{n}\right)$ is $\Omega\left(\sum_{i=1}^{n}\left|a_{i}\right|\right)$, where $\left|a_{i}\right|$ is the length of command sequence $a_{i}$.

${ }^{2} \mathrm{~A}$ single parameterized Wait(early-process-termination, $\mathrm{k}$ ) command could have been used. We chose the non-parameterized encoding version since it simplifies our proofs. 
PROOF SKETCH. The proof is very similar to that of Lemma 4 and, likewise, uses Observations 5 and 6. The proof of Observation 5 works for the DSM model without change. Observation 6 holds trivially in the DSM model, since each register is local to a single process and remote to all others. The rest of the proof is identical, except that we should now also prove that the addition of Wait(early-process-termination) commands does not not violate the claim. This follows easily from the fact that each Wait(earlyprocess-termination) added to $q_{j}$ 's code on account of an access by $q_{i}$, for some $i<j$, can be amortized against the first critical step by $q_{i}$ that accesses $q_{j}$ 's local segment.

THEOREM 9. Let $\left(A_{1}, \ldots, A_{n}\right)$ be order encoding DSM algorithms that satisfy weak obstruction-freedom. Then there is an execution $E \in \mathcal{E}$ with $\Omega(n \log n) R M R$ cost.

\section{APPLICATIONS}

\subsection{Mutual Exclusion}

The mutex object supports two operations: Enter and Exit. The Enter operation allows a process to enter the critical section; after completing the critical section, a process invokes an Exit operation. Any implementation of a mutex object must meet the following requirements: (1) at most a single process can be at its critical section in any given time (mutual exclusion), (2) if some process invokes Enter then, eventually, some process enters the critical section (deadlock freedom), and (3) a process completes the Exit procedure in a finite number of steps (finite exit).

We show that the algorithms $A_{i}^{\prime}$, given in the pseudo-code below, are order encoding. Algorithms $A_{i}^{\prime}, 1 \leq i \leq n$ use one deadlock-free mutex object $M E$, implemented by algorithms $A_{1}, \ldots, A_{n}$, a shared register counter initialized to 0 , and local register $\ell_{i}$.

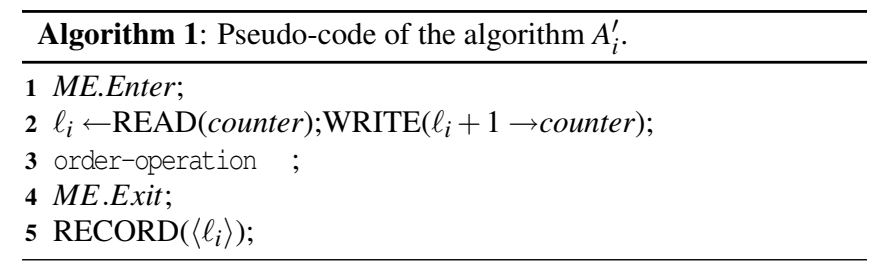

\section{LEMMA 10. Algorithms $A_{1}^{\prime}, \ldots, A_{n}^{\prime}$ are order-encoding.}

PROOF. Let $\pi \in S_{n}$ be a permutation and $L_{\pi}^{k}=\left(p_{\pi(1)}, \ldots, p_{\pi(k)}\right)$. To prove property (a) of Definition 1 , note that, in all executions, the $i$-th process entering the critical section records value $i$. Thus, in the $L_{\pi}^{k}$-solo execution $E_{\text {solo }}\left[L_{\pi}^{k}\right]$, process $p_{\pi(i)}$ records value $i$, for $1 \leq i \leq k$. Now let $1 \leq i<j \leq k$. In an execution $E$, where $p_{\pi(j)} \prec_{E}$ $p_{\pi(i)}$, process $p_{\pi(j)}$ enters the critical section before $p_{\pi(i)}$ does and so records a smaller value than is recorded by $p_{\pi(i)}$. Hence, $\vec{R}[E] \neq$ $\vec{R}\left[E_{\text {solo }}\left(L_{\pi}^{k}\right)\right]$.

To prove property (b), note that in any execution in which the processes $p_{\pi(1)}, \ldots, p_{\pi(k)}$ participate, the record vector is a permutation of $\{1, \ldots, k\}$. Thus, if the record vectors of two executions $E$ and $E^{\prime}$ in which the processes $p_{\pi(1)}, \ldots, p_{\pi(k)}$ participate differ, then they must differ in at least two components.

The definitions imply that a deadlock-free mutual-exclusion implementation satisfies weak obstruction-freedom, and hence, so do algorithms $A_{1}^{\prime}, \ldots, A_{n}^{\prime}$. We get:

THEOREM 11. Any deadlock-free implementation of mutual exclusion from read, write, and conditional operations has an execution whose RMR cost is $\Omega(n \cdot \log n)$.
Proof. Since algorithms $A_{1}^{\prime}, \ldots, A_{n}^{\prime}$ satisfy weak obstructionfreedom, by Lemma 10 and Theorem 1 , there is an execution $E^{\prime}$ of $A_{1}^{\prime}, \ldots, A_{n}^{\prime}$ with an RMR cost of $\Omega(n \cdot \log n)$. Let $E$ be the execution obtained from $E^{\prime}$ by removing all the steps performed by $A_{1}^{\prime}, \ldots, A_{n}^{\prime}$ that are not performed by $A_{1}, \ldots, A_{n}$. In $E^{\prime}$, each process performs at most 2 RMRs more than it does in $E$. It follows that $E$ is an execution of $A_{1}, \ldots, A_{n}$ whose RMR cost is $\Omega(n \cdot \log n)$.

\subsection{Bounded Counters}

A $b$-bounded counter is an object that supports the operations Increment and Reset. The object stores a value in $\{0, \ldots, b\}$ and is initialized to 0 . The operation Increment atomically increments the value of the object, unless it already has value $b$ (in which case the value is not changed), and returns its previous value. The operation Reset resets the value of the object to 0 and returns the previous value. Using our lower bound technique, we show that the RMR cost of calling Increment and Reset once is $\Omega(n \cdot \log n)$.

We prove that the algorithms $B_{i}$, given in pseudo-code below, are order-encoding. Algorithms $B_{i}, 1 \leq i \leq n$, use a single $b$-bounded counter object $B C$, for some $b \geq 2$, a shared integer counter initialized to 0 , and registers $\ell_{i}$ and $r_{i}$.

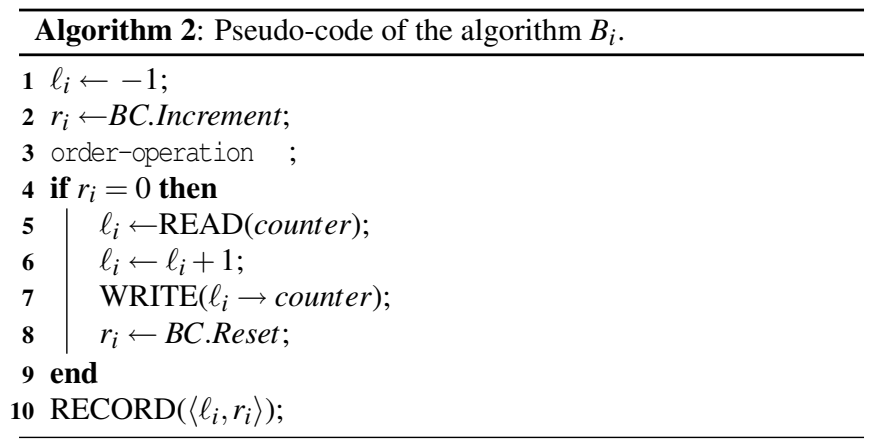

\section{LEMMA 12. Algorithms $B_{1}, \ldots, B_{n}$ are order-encoding.}

PROOF. Let $\pi \in S_{n}$ be a permutation, let $L_{\pi}^{k}=\left(p_{\pi(1)}, \ldots, p_{\pi(k)}\right)$ and let $E$ be an execution in which only the processes $p_{\pi(1)}, \ldots, p_{\pi(k)}$ participate. Clearly, in $E_{\text {sol }}\left(L_{\pi}^{k}\right)$ participating processes obtain response 0 from the Increment operation in line 3 and then obtain response 1 from the Reset operation in line 8. It follows that the value recorded by process $p_{\pi(j)}$ in $E_{s o l}\left(L_{\pi}^{k}\right)$ is $\langle j, 1\rangle$, for $j=1, \ldots, k$.

We now prove Property (a) of Definition 1 . Let $E$ be an arbitrary execution and $1 \leq i<j \leq k$. For the purpose of a contradiction assume that $p_{\pi(j)} \prec_{E} p_{\pi(i)}$, and that the record vector of $E$ is the same as that of $E_{s o l}\left(L_{\pi}^{k}\right)$. Then the Increment operation must return 0 for both processes, $p_{\pi(i)}$ and $p_{\pi(j)}$, or otherwise one of them would record $\langle-1, x\rangle$ for $x \in\{1, \ldots, b\}$. But then $p_{\pi(i)}$ cannot execute its order-operation before $p_{\pi(j)}$ resets the counter in line 8 . Hence, if $p_{\pi(i)}$ records $\left\langle a_{1}, 1\right\rangle$ and $p_{\pi(j)}$ records $\left\langle a_{2}, 1\right\rangle$, then $a_{1}>a_{2}$. This is different from the record-vector produces by $E_{s o l}\left(L_{\pi}^{k}\right)$, where $p_{\pi(i)}$ records $\langle i, 1\rangle$ and $p_{\pi(j)}$ records $\langle j, 1\rangle$.

To prove Property (b), assume that in execution $E$ the record $\langle x, y\rangle$ of process $p_{\pi(k)}$ is different from $\langle k, 1\rangle$. We show that there exists another process $p_{\pi(j)}, j \neq k$, whose record is different from $\langle j, 1\rangle$. If $x=-1$, then process $p_{\pi(k)}$ 's Increment operation in line 2 occurs after some other process $p_{\pi(j)}$ increments the counter $B C$ from 0 to 1 , and before $p_{\pi(j)}$ 's Reset operation in line 8 . Thus when that Reset occurs, then the counter has a value of $b$, and $p_{\pi(j)}$ 's record is 2 in the second component.

If $x \neq k$ and $x \neq-1, p_{\pi(k)}$ is the $x$-th process that executes line 6 , and not the $k$-th. Let $j=x$. Clearly, if process $p_{\pi(j)}$ executes line 
6 , then the first component of its record is not $j$, and if it does not execute line 6 , then the first component of its record is -1 .

Finally, assume that $x=k$ but $y \neq 1$. Since $x \neq-1$, process $p_{\pi(k)}$ increments the counter from 0 to 1 in line 2 , and no other process can reset it until $p_{\pi(k)}$ executes line 8 . Since $y \neq 1$, this Reset operation returns 2 (and thus $y=2$ ), and some other process $p_{\pi(j)}$ increments the counter after $p_{\pi(k)}$ executes line 2 and before $p_{\pi(k)}$ executes line 8 . But then $p_{\pi(j)}$ 's Increment operation returns 1 , and $p_{\pi(j)}$ 's record is $\langle-1,1\rangle$.

Since the algorithms $B_{1}, \ldots, B_{n}$ satisfy weak obstruction-freedom, we obtain with the same arguments as in the proof of Theorem 11:

THEOREM 13. Any weak obstruction-free implementation of a $b$-bounded counter, for $b>2$, from read and write operations has an execution in which each process calls Increment and Reset once, whose RMR cost is $\Omega(n \cdot \log n)$.

\subsection{Store/Collect Objects}

A one-shot (single-writer) store/collect object supports the operations Store and Collect. The object has $n$ components, initialized to $\perp$; each process can either store a value in its associated component or collect the values from all components; a collect should not miss a value of a preceding store nor include the value of a store that has not yet begun.

Formally, the object supports two operations: $\operatorname{Store}(v)$ by process $p_{i}$ makes $v$ be the value stored by $p_{i}$, while Collect() returns a vector $V$ of values, one for each process. If a Collect operation returns $V$ and $p_{i}$ is a process, then $V[i]=\perp$ only if no Store operation by $p_{i}$ completes before the Collect; if $V[i]=v \neq \perp$, then $v$ is the parameter of a Store operation $o p$ by $p_{i}$ that does not follow the Collect.

We use our lower bound technique, to prove that the RMR cost of calling store and collect once is $\Omega(n \cdot \log n)$.

We show that the algorithms given in pseudo-code below, are order-encoding. Algorithms $C_{i}, 1 \leq i \leq n$, use a single weak obstruction-free store/collect object, $C L$, and registers $\ell_{i}$ and $r_{i}$.

\begin{tabular}{ll}
\hline Algorithm 3: Pseudo-code of the algorithm $C_{i}$. \\
\hline $\mathbf{1} C L$. Store $(i)$; order-operation ; \\
$\mathbf{2} r_{i} \leftarrow$ CL.Collect; $\ell_{i} \leftarrow$ number of non- $\perp$ entries in $r_{i} ;$ \\
$\mathbf{3} \operatorname{RECORD}\left(\left\langle\ell_{i}\right\rangle\right) ;$
\end{tabular}

LEMMA 14. Algorithms $C_{1}, \ldots, C_{n}$ are order-encoding.

PROOF. Let $\pi \in S_{n}$ be a permutation, let $L_{\pi}^{k}=\left(p_{\pi(1)}, \ldots, p_{\pi(k)}\right)$ and let $E$ be an execution in which only the processes $p_{\pi(1)}, \ldots, p_{\pi(k)}$ participate. Clearly, in $E_{s o l}\left(L_{\pi}^{k}\right)$, process $p_{\pi(j)}, 1 \leq j \leq k$, collects non- $\perp$ values exactly from processes $p_{\pi(1)}, \ldots, p_{\pi(j)}$. Hence, the value recorded by process $p_{\pi(j)}$ in $E_{s o l}\left(L_{\pi}^{k}\right)$ is $\langle j\rangle$, for $j=1, \ldots, k$.

We now prove Property (a) of Definition 1 . Let $E$ be an arbitrary execution and $1 \leq i<j \leq k$, such that $p_{\pi(j)} \prec_{E} p_{\pi(i)}$; assume that $i$ and $j$ are the minimal indices with this property. Then process $p_{\pi(i)}$ reads a non- $\perp$ value from process $p_{\pi(j)}$, and since $i$ is minimal it follows that $p_{\pi(i)}$ reads non- $\perp$ values from $p_{\pi(1)}, \ldots, p_{\pi(i)}$.

To prove Property (b), assume that in execution $E$ process $p_{\pi(k)}$ records a value different than $k$, i.e., $p_{\pi(k)}$ misses the value stored by some process $p_{\pi(i)}, 1 \leq i<k$. The definition of a store / collect object implies that the collect of $p_{\pi(k)}$ starts before the store of $p_{\pi(i)}$ completes and hence before the collect of $p_{\pi(i)}$ starts. Let $p_{\pi(j)}$, $1 \leq j<k$, be the process whose collect starts last in $E$, then clearly, $p_{\pi(j)}$ records $k \neq j$, and Property (b) follows.

Since the algorithms $C_{1}, \ldots, C_{n}$ satisfy weak obstruction-freedom, we get in a manner similar to Theorem 11:
THEOREM 15. Any weak obstruction-free implementation of a store/collect object, from read, write and conditional operations has an execution in which each process calls Store and Collect once whose RMR cost is $\Omega(n \cdot \log n)$.

\section{SUMMARY}

We used a novel technique for proving lower bounds on the RMR cost for a variety of synchronization problems to derive an $\Omega(n \log n)$ lower bound for mutual exclusion, bounded counters and store/collect. Further research is to find additional applications for our technique or extend it to accommodate randomization and stronger primitives.

\section{Acknowledgements}

We would like to thank the referees for their helpful comments.

\section{REFERENCES}

[1] R. Alur and G. Taubenfeld. Results about fast mutual exclusion. RTSS 1992, pp. 12-21.

[2] J. Anderson and Y.-J. Kim. An improved lower bound for the time complexity of mutual exclusion. Dist. Comp., 15(4):221-253, 2002.

[3] J. Anderson, Y.-J. Kim, and T. Herman. Shared-memory mutual exclusion: Major research trends since 1986. Dist. Comp., 16:75-110, 2003.

[4] J. H. Anderson and Y.-J. Kim. Fast and scalable mutual exclusion. DISC 1999, pp. 180-194.

[5] J. H. Anderson and Y.-J. Kim. Adaptive mutual exclusion with local spinning. DISC 2000, pp. 29-43.

[6] J. H. Anderson and Y.-J. Kim. Nonatomic mutual exclusion with local spinning. PODC 2002, pp. 3-12.

[7] T. Anderson. The performance of spin lock alternatives for shared-memory multiprocessors. IEEE Transactions on Parallel and Distributed Systems, 1(1):6-16, 1990.

[8] R. Cypher. The communication requirements of mutual exclusion. SPAA 1995, pp. 147-156.

[9] R. Fan and N. Lynch. An $\Omega(n \log n)$ lower bound on the cost of mutual exclusion. PODC 2006, pp. 275-284.

[10] W. M. Golab, V. Hadzilacos, D. Hendler, and P. Woelfel. Constant-RMR implementations of CAS and other synchronization primitives using read and write operations. PODC 2007, pp. 3-12.

[11] M. Herlihy, V. Luchangco, and M. Moir. Obstruction-free synchronization: Double-ended queues as an example. ICDCS 2003, pp. 522-529.

[12] M. Herlihy and J. M. Wing. Linearizability: A correctness condition for concurrent objects. ACM Trans. Prog. Lang. Syst., 12(3):463-492, 1990.

[13] Y.-J. Kim and J. Anderson. A time complexity bound for adaptive mutual exclusion. DISC 2001, pp. 1-15.

[14] J. Mellor-Crummey and M. Scott. Algorithms for scalable synchronization on shared-memory multiprocessors. ACM Trans. Comput. Syst., 9(1):21-65, 1991.

[15] D. A. Patterson and J. L. Hennessy. Computer Organization and Design: The Hardware/Software Interface. Morgan Kaufmann, 1994.

[16] G. Taubenfeld. Synchronization Algorithms and Concurrent Programming. Prentice Hall, 2006.

[17] J.-H. Yang and J. Anderson. A fast, scalable mutual exclusion algorithm. Dist. Comp., 9(1):51-60, 1995. 